\title{
Zentralvorstandssitzung vom 17. Februar 2011
}

\begin{abstract}
Nachfolgeregelung Zulassungsstopp - Ende 2011 läuft der Zulassungsstopp aus. Die GDK hat sich für eine erneute Verlängerung um drei Jahre ausgesprochen. Der Zentralvorstand der FMH wird der Delegiertenversammlung antragen, entweder den Zulassungsstopp auslaufen zu lassen oder eine kantonale Regulierungsmöglichkeit für die künftige Ressourcensteuerung vorzuschlagen.
\end{abstract}

e-Health: Studie Regulierungsfolgenabschätzung Die FMH hat Kritik geübt an verschiedenen Punkten einer BAG-Studie zur Regulierungsfolgenabschätzung. Diese bezweckt, Kosten und Nutzen einer gesetzlichen Regulierung zu eHealth zu evaluieren. Das BAG bezieht die Stellungnahme der FMH in die Überarbeitung der Regulierungsfolgenabschätzung ein.

Ärztliche Medikamentenabgabe - FMH und santésuisse haben ein Absichtspapier zur ärztlichen Medikamentenabgabe unterschrieben, welches im März der Delegiertenversammlung vorgelegt wurde. Die konkreten Verhandlungen zum neuen Modell für die ärztliche Medikamentenabgabe beginnen im April.
Begleitforschung SwissDRG - Die FMH schreibt gemeinsam mit $\mathrm{H}+$ das Forschungsprojekt «Begleitende Untersuchung aus Anlass der Einführung von SwissDRG: Leistungs- und Kostenverschiebungen zwischen dem akutstationären und dem spital-/praxisambulanten Sektor» aus. Aktuell gibt es für diesen Bereich keine Statistiken.

Rare Diseases - Die FMH hat gemeinsam mit verschiedenen Gesundheitspartnern an der Kick-offSitzung zur Konstituierung der «IG Orphan Drugs» teilgenommen. Die Interessengemeinschaft beabsichtigt, die Sensibilisierungsarbeit für seltene Krankheiten in verschiedenen Bereichen zu koordinieren. Zunächst soll eine Liste seltener Krankheiten erarbeitet und eine Geschäftsstelle eingesetzt werden.

Arbeitsgruppe «Übergriff in Arztpraxen»-Die Arbeitsgruppe «Übergriff in Arzpraxen» hat die Arbeit aufgenommen. Ziel ist es, dass bereits registrierte und überführte Täter, die wieder ihre Tätigkeit in der Praxis aufnehmen, nicht erneut Übergriffe begehen.

\section{Wissen, was läuft.} Das News-Paket der FMH.

\author{
Schweizerische Ärztezeitung, Today's Press, \\ politoscope. Für Mitglieder kostenlos.
}

